\title{
Type of perception vs. lunge in fencing technique structure
}

\author{
Zbigniew BORYSIUK \\ Faculty of Physical Education and Physiotherapy at the Opole University of Technology (Poland)
}

\author{
5th IMACSSS World Scientific Congress Abstracts, Rio Maior (Portugal), October 6-8 \\ Section: Biological, kinesiological and physiological aspects of MA\&CS \\ Type: Oral communication
}

\section{Introduction}

The specific fencing duel is based on fencers' response on the three particular kinds of stimuli such as: visual, kinesthetic and acoustic. Functional efficiency of senses which are responsible for perception processes is the main condition of gaining achievement in sports. Moreover the above mentioned analyzers are the significant elements to excellence fencing techniques. Although, visual perception is not the only factor playing an important role when trying to anticipate the contestants' intentions. Many of actions undertaken during a fencing fight (e.g., cuts, binds and parries) require high level of kinesthetic feeling in other words appropriate responses on tactile stimulation. Significant part of actions' preparation depends on mastering sense of rhythm during the fight which should be followed by acoustic stimuli perception from foot work and technique actions performed by fencing weapon (Hassan \& Klauck, 1998; Do \& Yiou, 1999). Expression of different stimuli which is observed on the training and competitive surroundings demand particular cautions and the ability of attention divisibility from fencers. The results coming from various experiments prove that athletes respond on tactile stimuli in comparison with visual stimuli in the shortest time. The differentiation is estimated approximately from 100 to $120 \mathrm{~ms}$. We observe slight variation in reference to information processes in response to acoustic and tactile stimuli to gain 10-15 ms (Borysiuk \& Waśkiewicz, 2008).

\section{Methodology}

Six female participants mainly junior epeeists represented Silesian Macroregion. Competitors performed consecutive six efforts in the form of simple attack by lunge: three on the established by the trainer step forward (without blades contact) and three on the "letting go" of the competitor's blade by the coach. The goal of the investigation was to consider a dynamic structure of fencing lunge through the establishment of muscle activation order: rectus femoris and biceps femorisin a forwarding leg, musculus gastrocnemius in back leg, biceps brachii, triceps brachii and brachioradialis in a weapon maintaining arm. Moreover, we measured medium value of bioelectric muscle tension (EMG). In research procedures we applied 16 channel EMG Noraxon telemetric device registering muscle activity in agreement to recommendation by SENIAM. Surface electromyography system was synchronized with speed camera (Point Grey Gazelle) and additionally with software ProAnalysis.

\section{Results}

Firstly, while performing simple attack on the coach signal which was established with his forwarding step treated as the visual stimulus the initial muscle which is musculus triceps brachii of dominated hand was activated in response. Secondly, the activation of musculus gastrocnemius (captuslateralis) of the back leg appeared. Thirdly, the stimulation of musculus gastrocnemius (captusmedialis) and rectus femoris of the forwarding leg was observed. Then an initial stimulation

*Email: z.borysiuk@po.opole.pl 
of a weapon maintaining arm muscles was recorded as the following: brachioradialis and biceps brachii. After that a rectus femoris of the forwarding leg was stimulated. While the simple attack with lunge when coach provided a signal by breaking a contact between weapon blades (tactile stimulation) a simultaneous activation of musculus gastrocnemius (captuslateralis and captusmedialis) of the back leg was initiated. In the next order triceps brachii of weapon maintaining arm and then rectus femoris of forwarding leg were followed by brachioradialis and biceps brachiiof dominated arm were stimulated. Finally, biceps femoris of the back leg activation was registered.

\section{Discussion and conclusion}

The consecutive of particular muscle activation during simple thrust with lunge performing depends on the type of stimulus in which a fencer reacts. Classical rules of fencing technique say that attack should be initiated by extending arm are confirmed only in a simple reaction on a visual stimulus. In this case triceps brachii was the first muscle to be activated as an extensor of elbow joint. On the other hand, if movement was initiated by tactile stimulation (allowing a contact with competitor's blade) an activation of musculus gastrocnemius appears as first (captuslateralis and captusmedialis). Rectus femoris of the back leg as an extensor of knee joint and a flexor of hip-joint in both trials was activated as the third one. The mentioned phenomenon does not confirm the generally accepted theory concerning the sequence of movement of particular limbs while performing a simple thrust with lunge. Medium value of bioelectric muscle tension (EMG) generated in time of performing a simple attack with lunge also depends on different kinds of stimulus. In reference to a visual stimulus, the EMG signal manifested lower level of bioelectrical tension when we compare the same parameters in response to a tactile stimulus. An analysis of EMG signal indicates that investigated fencers generate higher muscle tension when focusing on tactile stimulus when compared to stimulation of visual receptors (Borysiuk, 2009). The concept mentioned above confirms that the preparation period while fencers anticipate visual stimuli decreases the value of EMG signal. Considering conclusions presented above can be applied in the initial stage of learning processes. Coaches should implicate appropriate methods of teaching various techniques based on shaping different kinds of perception. One of the major tasks which is undertaken by fencers during the lesson with trainer is mastering of selecting an adequate time to start an offensive action. Fencers in professional training process acquire new movement patterns and technical-tactical skills which can be used in actual fighting conditions. These skills involve simple reaction time in response to tactile and visual stimuli. The types of reactions are manifested in practice as sensori-motor responses and should be trained by simple and compound exercises according to motor learning and performance scheme introduced in Czajkowski (2011).

\section{References}

Hassan, E.A., \& Klauck, J. (1990). Kinematics of Lower and Upper Extremity Motions During the Fencing Lunge: results and training impications. "Deutsche Sporthoschule". Koln. Germany ISBS 98-Procedings II, s. 171.

Do, M.C., \& Yiou, E. (1999). Do centrally programmed anticipatory postural adjustments in fast stepping affect performance of an associated "touche" movement?. Experimental Brain Research, 129(3), 462-466.

Borysiuk, Z., \& Waśkiewicz, Z. (2008). Information Processes, Stimulation and Perceptual Training in Fencing. Journal of Human Kinetics, 19, 63-82.

Borysiuk, Z. (2009). Modern Saber Fencing. "SKA Sword Play Books". Staten Island. New York 10314, s. 235.

Czajkowski, Z. (2011). Directing the process of training, taking competition and fencer's personality dimensions as a model. IDO-Movement for Culture. Journal of Martial Arts Anthropology, 11(4), 48-56.

Key words: EMG signal; tactile stimulation; advance actions; combat sports; fencing. 